\title{
Aggressive repeat surgery for focally recurrent primary glioblastoma: outcomes and theoretical framework
}

\author{
Michael E. Sughrue, MD, ${ }^{1}$ Tyson Sheean, MBBS, ${ }^{2}$ Phillip A. Bonney, BS, ${ }^{1}$ Adrian J. Maurer, BA, ${ }^{1}$ \\ and Charles Teo, MBBS, FRACS ${ }^{2}$
}

\begin{abstract}
${ }^{1}$ Department of Neurosurgery, University of Oklahoma Health Sciences Center, Oklahoma City, Oklahoma; and ${ }^{2}$ The Centre for Minimally Invasive Neurosurgery, Prince of Wales Private Hospital, Sydney, Australia
\end{abstract}

\begin{abstract}
OBJECT The relative benefit of repeat surgery for recurrent glioblastoma is unclear, in part due to the very heterogeneous nature of the patient population and the effect of clinician philosophy on the duration and aggressiveness of treatment. The authors sought to investigate the role of time to last recurrence on patient outcomes following aggressive repeat surgery for recurrent glioblastoma.

METHODS The authors present outcomes in 104 patients undergoing repeat surgery for focally recurrent glioblastoma with at least $95 \%$ resection and adjuvant treatment at most recent prior surgery. In addition to common variables, they provide data regarding the period of progression-free survival (PFS) following an aggressive lesionectomy for focally recurrent primary glioblastoma (T2) and the time the tumor took to recur since the previous surgery (T1). They term the ratio $\mathrm{T} 1 / \mathrm{T} 2$ the relative aggressivity index (RAI).

RESULTS The median PFS was 7.8 months, 6.0 months, and 4.8 months following the second, third, and fourth-sixth craniotomies, respectively. Importantly, there was a wide range of outcomes, with time to postoperative recurrence ranging from 1 to 24 months in this group. Analysis showed no meaningful relationship between T1 and T2, meaning that previous PFS is entirely unable to predict the PFS that another surgery will provide the patient.

CONCLUSIONS Repeat surgery for glioblastoma is beneficial in many cases, however this is hard to predict preoperatively. Often, surgery can provide the patient with a good period of disease freedom, but this is variable and in general it is not possible to reliably predict who these patients are.

http://thejns.org/doi/abs/10.3171/2014.12.FOCUS14726
\end{abstract}

KEY WORDS surgery; glioblastoma; astrocytoma; GBM; recurrent glioma; recurrence; survival

$\mathrm{R}$ ECURRENCE in glioblastoma (GBM) is inevitable. Given that GBMs typically recur focally, ${ }^{8}$ and in many cases, resection of all or most of the enhancing portion of the tumor is possible in experienced hands, 5,6 repeat resection is often a feasible management strategy. However, there is a paucity of literature to inform decision making for resectable focally recurrent tumor and help clinicians and surgeons decide when surgery is worth the risk.

That our opinions and actions inherently change the overall survival (OS) independent of the intervention being studied is rarely accounted for in GBM studies using survival as an end point. This phenomenon is not unique to GBM; the effect of clinician mindset on outcomes has been described in other neurological diseases. ${ }^{2}$ This effect complicates our interpretation of data for this disease and inherently creates a big, unspoken confounder in clinical GBM research: the effect of repeat surgery on survival. It is implausible that good clinical trials using OS as the primary end point can be performed when some patients receive 4 craniotomies for tumor resection and others receive 1 , without a serious effort to address this issue in a scientific fashion. Removing gross tumor volume inherently "resets the clock," even if for a short time, and doing 4 repeat surgeries likely significantly alters OS compared with doing nothing at the time of first recurrence, for it would contradict intuition to suggest that removing bil-

ABBREVIATIONS GBM = glioblastoma; KPS = Karnofsky Performance Status; OS = overall survival; PFS = progression-free survival; RAI = relative aggressivity index (T1/T2 ratio); RANO = Response Assessment in Neuro-Oncology; T1 = time from previous surgery to GBM recurrence; T2 = period of progression-free survival following an aggressive lesionectomy for focally recurrent primary GBM.

SUBMITTED October 24, 2014. ACCEPTED December 12, 2014.

INCLUDE WHEN CITING DOI: 10.3171/2014.12.FOCUS14726

DISCLOSURE Dr. Teo is a consultant for Aesculap; however, this work does not directly relate to the content of this report. The authors declare that they are not involved in any other relationships with companies that make products related to this study. 
lions of cancer cells accomplishes nothing., ${ }^{1,47}$ The more meaningful questions, then, are what this accomplishes and whether it is worth the risk.

Recurrent GBM is frustratingly difficult to study with scientific rigor, in large part because of the seemingly endless heterogeneity of the patient population. Patients have often been treated with multiple previous adjuvant therapies (often including experimental agents of unknown effect), have received resections of differing aggressiveness, and have recurrences in anatomical locations with differing surgical risks. By using OS as an end point for patients with variably aggressive diseases and by offering repeat surgery based on variable criteria, we have set up all clinical trials in recurrent GBM for failure by underpowering them for the extremely heterogeneous population they are studying, a limitation impossible to overcome through randomization.

This study attempts to make inroads into this difficult problem by presenting data regarding the value of aggressive repeat lesionectomy surgery for a relatively homogeneous patient population with focally recurrent primary GBM after initial treatment with aggressive $(>95 \%)$ resection, fractionated radiotherapy, and temozolomide monotherapy. This provides data regarding outcomes from patients treated under the philosophy that, until a truly curative adjuvant therapy is identified, the goal of adjuvant therapies should be to space out the recurrences such that repeat surgery is more acceptable. We have met few GBM patients who, when faced with their prognosis, would object to repeat resections followed by additional adjuvant treatment every 2 years if such an interval could be achieved. Given the inherent challenges of this disease, a strategy that transforms it into a chronic medical condition would be a welcome improvement.

Attacking the problem from this viewpoint, we introduce an easily derived variable, which we term the relative aggressivity index (RAI). The conceptual basis of the RAI is depicted in Fig. 1. In short, the RAI is calculated as the ratio of the previous progression-free interval (T1) to the most recent progression-free interval (T2). By this definition, an RAI less than 1.0 indicates a longer time to the most-recent recurrence (T2) than to the next-most-recent recurrence (T1), and conceptually this suggests a clinically less aggressive tumor at present. Conversely, a patient with a relatively short T2 will have an RAI greater than 1.0 , which suggests that the tumor has become more aggressive. The RAI could also be mathematically expressed as $T_{n-1} / T_{n}$ or $P_{F-1} / P_{n F}$, where $n$ is the craniotomy being assessed. However, for simplicity, we will denote T2 as the most-recent PFS and T1 as the next-most-recent PFS. In our initially optimally treated cohort, we demonstrate the spectrum of RAIs and study this variable's relationship to other known prognostic factors.

\section{Methods \\ Patient Population}

This is a retrospective review based on all cases in which recurrent primary GBM was surgically treated at the Prince of Wales Hospital in Sydney, Australia, between 1995 and 2009. To limit heterogeneity, we excluded all patients who underwent previous confounding treatments and limited our analysis to those who had received previous aggressive resection of at least $95 \%$ of the tumor volume, followed by adjuvant fractionated radiotherapy and temozolomide chemotherapy after initial surgery. Patients undergoing repeat surgery for markedly incompletely resected tumors and patients with secondary GBMs were excluded in order to reduce cohort heterogeneity. We also excluded patients who underwent surgery for imaging findings that were found on histological analysis to represent only treatment effect, without evidence of viable tumor cells.

All patients underwent postoperative MRI in the first 48 hours after surgery, at 6 weeks postsurgery, and then every 2-3 months if they remained asymptomatic. Repeat imaging was performed earlier if the patient complained of new or worsening neurological deficits, worsening headaches, or if indicated for any other reason. Extent of resection was determined from the first postoperative imaging study using computerized volumetric analysis as described below. This study was performed with the approval of the Prince of Wales Hospital ethics committee, and complied with local institutional guidelines for human research.

\section{Management Paradigm and Surgical Technique}

All surgeries were performed using minimally invasive keyhole techniques tailored to the exact pathoanatomic configuration of the patient's tumor. A holistic description of strategies for planning keyhole approaches to these large tumors is beyond the scope of this paper, but in short, we attempt to expose as little brain as possible while still having enough room to achieve the goals of surgery. Involved vessels are skeletonized, and as much tumor as possible is removed from them. Resection proceeds until all tumor is removed or we encounter the internal capsule or presumed motor region. The goal of all surgeries was $>$ 95\% resection.

Postoperatively, patients are weaned off steroids in 2-5 days as tolerated. Discharge is planned for the 1st or 2 nd postoperative day. Patients with neurological deficits are immediately started on aggressive physical, occupational, or speech therapy on an inpatient or outpatient basis. We do not prophylactically treat patients without a seizure history with antiepileptics, and whenever clinically possible, patients who require antiepileptic medication are treated with non-enzyme-inducing drugs.

\section{Data Collection}

Extent of resection was calculated using volumetric assessments. The total planimetric tumor volume on all slices was first calculated as a percentage of total volume to normalize values between imaging studies. Definitions of tumor volumes and recurrence were made using the criteria suggested by the Response Assessment in Neuro-Oncology (RANO) Working Group. ${ }^{9}$ Tumor and total brain volumes for each image were analyzed using identical scale, brightness, and contrast scanning and were calculated for analogous brain regions between imaging studies. Tumor and brain outlines were traced using Adobe Photoshop, and overlays of tracings were exported and quantitatively assessed using Image $(\mathrm{NIH})$. 


\section{Relative Aggressivity Index $(\mathrm{RAI})=\mathrm{T} 1 / \mathrm{T} 2=\mathrm{PFS}_{1} / \mathrm{PFS}_{2}$}
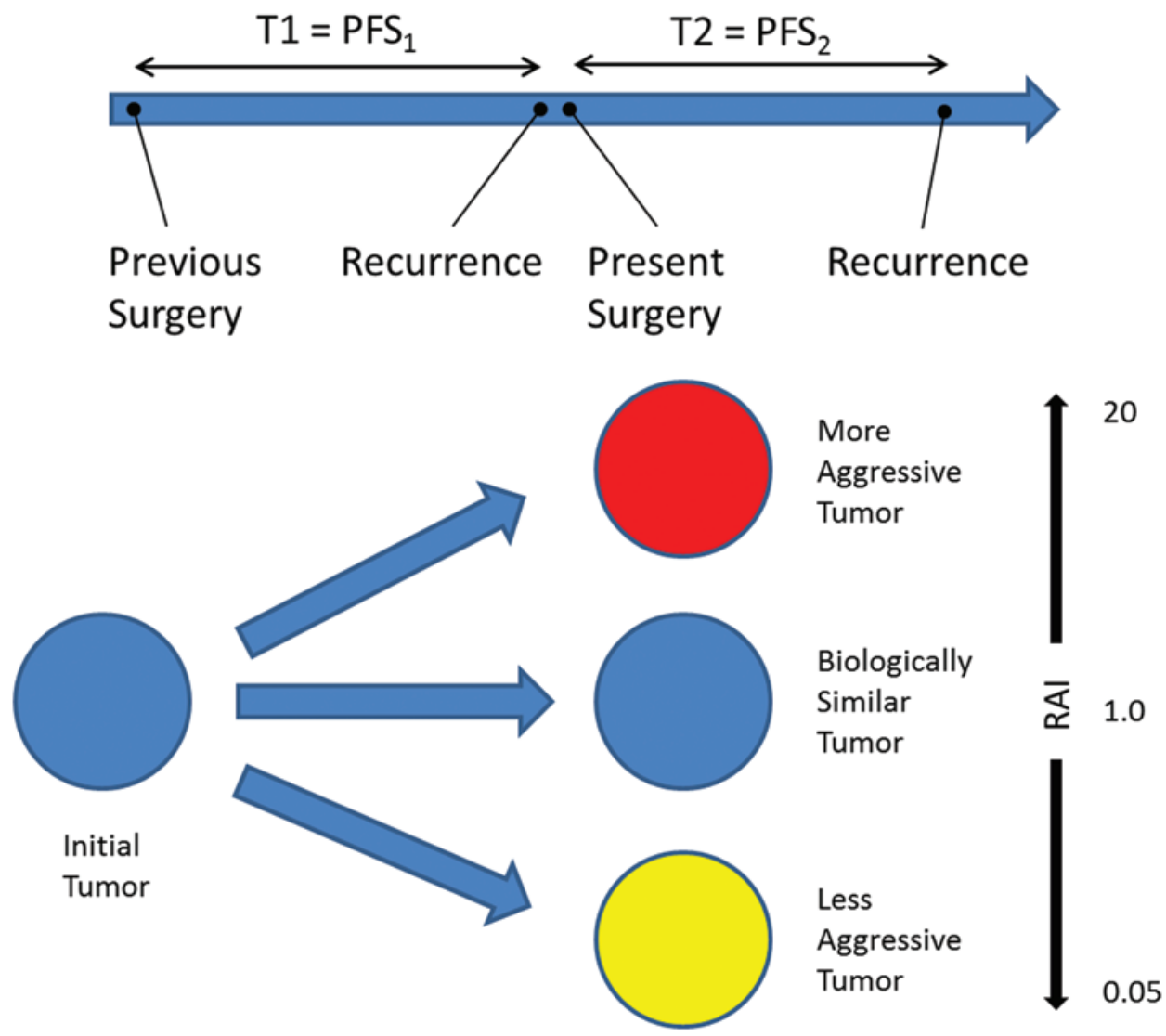

FIG. 1. Timeline demonstrating the definitions of $\mathrm{T} 1$ and $\mathrm{T} 2$ as used in this study (upper) and schematic demonstrating a potential interpretation of the RAI (lower).

Recurrence was defined using RANO criteria; thus a significant increase in the volume of T2 signal hyperintensity was considered recurrence. ${ }^{9}$ Progression-free survival was defined as the time between an operation and the date of documented evidence of recurrence or clinical deterioration. Given that these patients all had completed chemoradiation therapy prior to repeat surgery, using clinical deterioration as an evidence of recurrence is in line with RANO criteria.

Neurological deficits were compared with the preoperative baseline; worsening of a preexisting deficit or the presence of a new neurological deficit were both considered neurological complications. Deficits were considered permanent if they persisted past 6 months postoperatively or until the last follow-up visit, whichever came first. The term "surgical complication" includes CSF leak, meningitis, and wound infection or breakdown. Central pathology review was performed on the basis of the most recent WHO guidelines. ${ }^{3}$ All clinical assessments were performed by a neurosurgeon.

\section{Statistical Analysis}

The relationship between patient demographic characteristics was assessed using univariate analysis to iden- tify potential between-group differences. Binary variables were compared using Pearson's chi-square test. Continuous variables were compared using an independent-samples t-test or ANOVA. PFS analysis was performed using Kaplan-Meier method, where statistical significance was determined using log-rank test. The relationship between $\mathrm{T} 1$ and T2 was determined using simple linear regression. The interrelationship between potential confounding variables and RAI was explored using ANCOVA (analysis of covariance). Parametric tests were used after formally demonstrating normality of data. Continuous variables are presented as mean $\pm \mathrm{SE}$. Statistical tests were considered significant with $\mathrm{p}<0.05$ after correction for multiple comparisons.

\section{Results}

\section{Patient Population}

We identified 104 patients undergoing repeat surgery for primary GBM who had previously undergone $>95 \%$ resection and standard chemoradiation as described in the inclusion criteria. There were 59 patients undergoing their second resection, 24 undergoing a third resection, and 21 undergoing a fourth, fifth, or sixth resection. Thirty-two patients had frontal-lobe-predominant gliomas, 23 
patients had temporal-lobe-predominant gliomas, 20 patients had parietal-lobe-predominant gliomas, 10 patients had occipital-lobe-predominant gliomas, and 10 patients had multilobar insular-involving gliomas. The remaining patients had tumors centered in deep structures, such as the thalamus.

A total of $77(74 \%)$ of 104 patients received $>95 \%$ resection, and 21 of these patients $(20 \%$ of the whole group) received radiographic gross-total resection. Only 6 patients $(5.7 \%)$ received $<90 \%$ resection. Forty-seven patients underwent repeat treatment with temozolomide, 3 patients underwent bevacizumab therapy, 11 patients underwent adjuvant therapy with other alkylating agents (procarbazine, carboplatin), 5 patients underwent therapy with various experimental agents, and 38 patients underwent no adjuvant chemotherapy after repeat surgery.

\section{Progression-Free Survival After Repeat Surgery}

Progression-free survival data are shown in Table 1. Following a second craniotomy, the median PFS was 7.8 months. There was a wide range of outcomes, with time to postoperative recurrence ranging from 1 to 24 months. Twenty-three percent of patients experienced more than 14 months of PFS, while $12 \%$ had a recurrence within 3 months following surgery.

Following a third craniotomy, the median PFS was 6.0 months; however, again there was a wide range of outcomes, with time to postoperative recurrence ranging from 2 to 22 months. Twenty-nine percent of patients experienced more than 14 months of PFS, while $21 \%$ had a recurrence within 3 months following surgery.

Following a fourth, fifth, or sixth craniotomy, the median PFS was 4.8 months, with time to postoperative recurrence ranging from 1 to 13 months. Thirteen percent of patients experienced more than 14 months of PFS, while $27 \%$ had a recurrence within 3 months following surgery.

Kaplan-Meier analysis demonstrated no significant difference in PFS between patients undergoing second, third, or fourth-plus (i.e., fourth, fifth, or sixth) craniotomies (log-rank test; Fig. 2). Also, Kaplan-Meier analysis demonstrated no difference in PFS in patients treated with different classes of postoperative adjuvant therapies in this cohort, suggesting that the intragroup variability in PFS cannot be easily explained by differences in adjuvant therapies (Fig. 2).

TABLE 1. Most recent PFS stratified by craniotomy number

\begin{tabular}{cccc}
\hline & \multicolumn{3}{c}{ Craniotomy Number } \\
\cline { 2 - 4 } Variable & 2 & 3 & $4-6$ \\
\hline No. of patients & 59 & 24 & 21 \\
\hline PFS & & & \\
\hline$>14$ mos & $23 \%$ & $29 \%$ & $13 \%$ \\
\hline $6-14$ mos & $35 \%$ & $17 \%$ & $20 \%$ \\
\hline $3-6$ mos & $30 \%$ & $33 \%$ & $40 \%$ \\
\hline$<3$ mos & $12 \%$ & $21 \%$ & $27 \%$ \\
\hline Median PFS & $7.8 \mathrm{mos}$ & $6.0 \mathrm{mos}$ & $4.8 \mathrm{mos}$ \\
\hline PFS range & $1-24 \mathrm{mos}$ & $2-22 \mathrm{mos}$ & $1-13 \mathrm{mos}$ \\
\hline
\end{tabular}
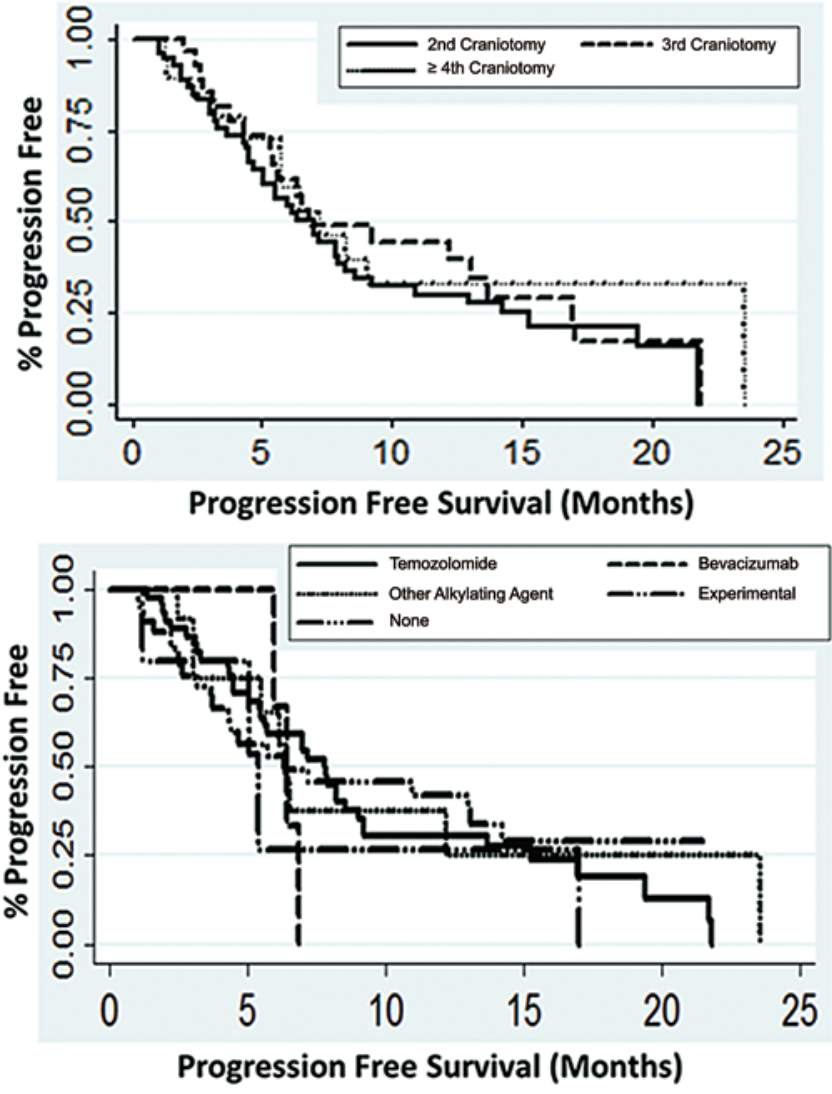

FIG. 2. Kaplan-Meier survival plots demonstrating significant univariate predictors of overall postoperative survival in this study. Plots demonstrate survival stratified by 2nd, 3rd, or 4th-plus craniotomy (upper) and postoperative adjuvant chemotherapy (lower).

\section{The RAI: Overall Outcomes and Analysis of Confounders}

Figure 3 demonstrates linear regression analysis studying the relationship between the time since previous resection (T1, x-axis) and the PFS after our surgery (T2, y-axis). We found no meaningful relationship between $\mathrm{T} 1$ and $\mathrm{T} 2$, meaning that the time it took the tumor to recur since the

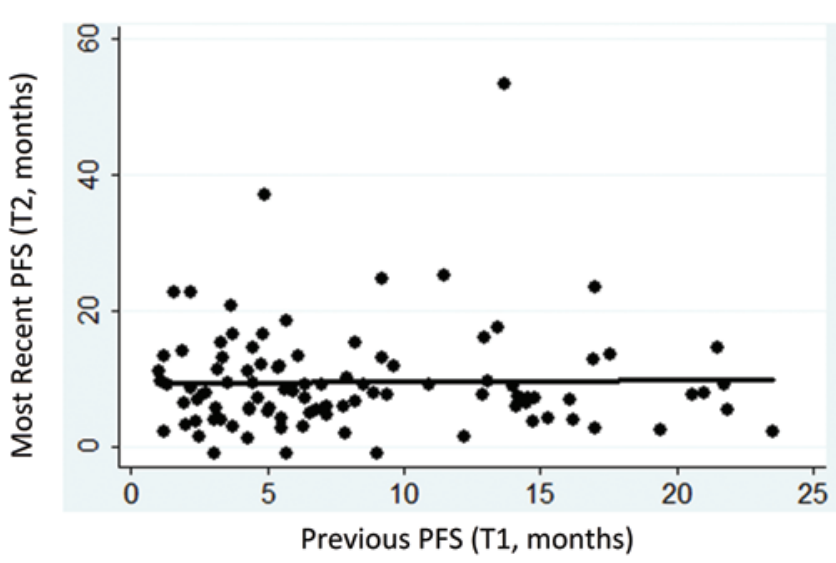

FIG. 3. A scatterplot and regression line demonstrating the relationship between most recent PFS (T2) and next most recent PFS (T1). 
last operation is entirely unable to predict the time free of radiographic or clinical evidence of tumor recurrence the present surgery will provide the patient. We also performed linear regression analyses relating initial PFS to third PFS and fourth-plus PFS, and similarly, we found no correlation between these variables.

Given this surprising finding, we have summarized the RAI (T1/T2) values for patients undergoing second, third, and fourth-plus craniotomy in Table 2 in an attempt to understand the RAI outcomes that patients exhibit following repeat operations. We found that RAI values are quite similar for patients undergoing second or third craniotomy and slightly less favorable for patients undergoing a fourth, fifth, or sixth craniotomy (median RAI for second craniotomy 1.28 , for third craniotomy 1.31 , for fourth-plus craniotomy 1.51). Again, this ratio was highly variable, ranging from 0.10 to 33.3 , meaning that patients could experience recurrence in $3 \%$ of the time to the last recurrence, or be recurrence-free 10 times as long as after the previous surgery. This is consistent with the idea that $\mathrm{T} 2$ has no relationship to $\mathrm{T} 1$, or in other words, that time to the last recurrence cannot be used to predict what a repeat surgery will accomplish. Forty-two percent of patients undergoing a second or third craniotomy had a longer time to next recurrence than after their previous surgery, as did $29 \%$ of patients undergoing a fourth, fifth, or sixth craniotomy.

Tables 3-6 demonstrate RAI outcomes grouped by tumor location, extent of resection at last surgery, Karnofsky Performance Status (KPS), and postoperative adjuvant therapy to determine whether the interpatient variance could be explained by potential prognostic confounding variables. As the tables demonstrate, none of these factors significantly influenced RAI on univariate analysis.

\section{Surgical Complications}

Three patients experienced wound infections, all after the third craniotomy ( $2.9 \%$ rate of infections after all repeat craniotomies for GBM). Two of these patients received bevacizumab after surgery. Two patients required treatment for CSF leak (1.9\% rate of CSF leak for all patients). New or worsened arm, leg, or face weakness occurred in 19 patients (18\%) and improved in many cases. Eight cases of weakness occurred with second resection (14\%), 7 with third (29\%), and 4 with fourth-plus (19\%).

TABLE 2. RAI stratified by craniotomy number

\begin{tabular}{cccc}
\hline & \multicolumn{3}{c}{ Craniotomy Number } \\
\cline { 2 - 4 } Variable & 2 & 3 & $4-6$ \\
\hline No. of patients & 59 & 24 & 21 \\
\hline RAl & & & \\
\hline$<1.0$ & $42 \%$ & $42 \%$ & $29 \%$ \\
\hline $1.0-2.0$ & $24 \%$ & $25 \%$ & $24 \%$ \\
\hline $2.0-5.0$ & $20 \%$ & $29 \%$ & $19 \%$ \\
\hline$>5.0$ & $14 \%$ & $4 \%$ & $29 \%$ \\
\hline Median RAI & 1.28 & 1.31 & 1.51 \\
\hline RAl range & $0.10-10.3$ & $0.13-5.9$ & $0.25-33.3$ \\
\hline
\end{tabular}

TABLE 3. RAI stratified by tumor location

\begin{tabular}{lccccc}
\hline \multirow{2}{*}{ Variable } & \multicolumn{5}{c}{ Predominant Tumor Location } \\
\cline { 2 - 6 } & Temporal & Parietal & Occipital & MIG & Frontal \\
\hline No. of patients & 23 & 20 & 10 & 10 & 32 \\
\hline RAI & & & & & \\
\hline$<1.0$ & $57 \%$ & $30 \%$ & $40 \%$ & $40 \%$ & $38 \%$ \\
\hline $1.0-2.0$ & $4 \%$ & $40 \%$ & $20 \%$ & $30 \%$ & $34 \%$ \\
\hline $2.0-5.0$ & $26 \%$ & $20 \%$ & $20 \%$ & $30 \%$ & $19 \%$ \\
\hline$>5.0$ & $13 \%$ & $10 \%$ & $20 \%$ & $0 \%$ & $9 \%$ \\
\hline Median RAI & 0.82 & 1.39 & 1.33 & 1.25 & 1.14 \\
\hline
\end{tabular}

MIG = multilobar insular-involving glioma .

Speech disturbance occurred in 5 patients (9\% of leftsided cases, $4.8 \%$ of all cases) - after second resection in 4 cases and after third resection in 1 case. There was no difference in rates of weakness (14\% vs $24 \%, p=0.20$, Fisher's exact test) or overall complications ( $22 \%$ vs $36 \%$, $\mathrm{p}=0.19$, Fisher's exact test) between the second craniotomy and third-plus craniotomy groups.

\section{Discussion}

The ideologies underpinning clinicians' philosophies on when to stop offering repeat surgery are largely based on experience, anecdotal conjecture from colleagues, personal opinions regarding the relative utility of surgery and/or other adjuvant treatments, and most importantly, their views regarding the risk-benefit ratio of aggressive, multimodality therapy for GBM. While strict indications for repeat surgery are poorly addressed in the literature, at aggressive centers, a common approach is to offer surgery to patients with surgically resectable, focally recurrent tumors, a good KPS score, and a long progression-free period since their last surgery. This last criterion is included in part because performing a repeat resection after a short interval between recurrences is viewed as putting the patient through the rigor of repeated operations without a sufficient recovery period.

The standard treatment goal of GBM therapy has been to lengthen OS; however, OS can typically be lengthened by repeatedly resecting the recurrent tumors as they are detected, even if the increase is only modest. Thus, OS in

TABLE 4. RAI stratified by extent of resection at most recent surgery

\begin{tabular}{lcccc}
\hline \multirow{2}{*}{ Variable } & \multicolumn{4}{c}{ Percentage Resection at Most Recent Operation } \\
\cline { 2 - 5 } & $100 \%$ & $95-99 \%$ & $90-94 \%$ & $<90 \%$ \\
\hline No. of patients & 21 & 56 & 13 & 6 \\
\hline RAI & & & & \\
\hline$<1.0$ & $24 \%$ & $45 \%$ & $62 \%$ & $33 \%$ \\
\hline $1.0-2.0$ & $33 \%$ & $29 \%$ & $8 \%$ & $17 \%$ \\
\hline $2.0-5.0$ & $38 \%$ & $18 \%$ & $15 \%$ & $33 \%$ \\
\hline$>5.0$ & $5 \%$ & $9 \%$ & $15 \%$ & $17 \%$ \\
\hline Median RAI & 1.56 & 1.14 & 0.76 & 1.25 \\
\hline
\end{tabular}


TABLE 5. RAI stratified by KPS score at last surgery

\begin{tabular}{ccccc}
\hline & \multicolumn{4}{c}{ KPS Score } \\
\cline { 2 - 5 } Variable & $90-100$ & $70-80$ & 60 & $<60$ \\
\hline No. of patients & 22 & 42 & 22 & 11 \\
\hline RAI & & & & \\
\hline$<1.0$ & $45 \%$ & $45 \%$ & $27 \%$ & $45 \%$ \\
\hline $1.0-2.0$ & $23 \%$ & $33 \%$ & $23 \%$ & $9 \%$ \\
\hline $2.0-5.0$ & $18 \%$ & $19 \%$ & $18 \%$ & $27 \%$ \\
\hline$>5.0$ & $14 \%$ & $2 \%$ & $14 \%$ & $18 \%$ \\
\hline Median RAI & 1.27 & 1.14 & 1.82 & 1.14 \\
\hline
\end{tabular}

GBM is inherently a function of length of time between recurrences, and the patients' and clinicians' opinion of whether performing surgery that frequently is acceptable. In this framework, the goal of adjuvant therapies should be to extend the period between recurrences, such that repeat surgery is a more acceptable option.

In this study, we demonstrate that there is no meaningful relationship between the period of PFS following aggressive lesionectomy for focally recurrent primary GBM (T2) and the time to recurrence since the previous surgery (T1). In other words, we have a very limited ability to predict how long repeat surgery will keep the tumor at bay based on the patient's past experience. In many cases, the tumor takes as long or longer to re-recur, and more rapid recurrences are less common than we thought prior to reviewing our data. This suggests that many focally recurrent tumors return with roughly the same clinical behavior they exhibited following the prior surgery. In many cases, tumors are clinically less aggressive, suggesting some biological effect of adjuvant therapies, a better surgical resection, or at least that surgery removes some of the more biologically aggressive cell populations in some cases (reducing a tumor's RAI). Even after fourth and fifth craniotomies, some patients maintain a low RAI; however, consistent with our intuition, the proportion of low-RAI patients decreases over time.

This study also provides data describing the RAI, an easily calculated, rough measure of clinical aggressiveness of GBM. We did not set out to determine whether specific variables (such as individual adjuvant therapies or extent of tumor resection) improve time to recurrence, and the study clearly is not powered to evaluate these variables in a meaningful fashion-we only sought to determine if the variability of the RAI was an artifact created by these confounders. Our data suggest that RAI varies significantly between patients and varies independently of other known or potential confounding variables, such as differences in extent of resection, tumor location, and postoperative KPS, suggesting that it is a valid measure of clinical outcome in and of itself. By focusing on lengthening the intervals between operations, the use of RAI as an end point avoids the problematic confounding effect of repeat surgery on OS. Thus, if a candidate therapy is biologically effective against the tumor, yet the patient decides against any more surgery, this does not affect the overall assessment of the drug's utility any differently than if the patient went on to undergo 3 additional surgeries, radiosurgery, and temozolomide treatment. Additionally, the use of RAI as an end point potentially reduces the confounding effect of interpatient biological variability by normalizing growth kinetics to the historical behavior of the tumor. Further, within a given patient the biology of GBM will change substantially over time. By accounting for previous clinical course, a patient's RAI may thus better suggest the biological response of the tumor to treatment. For instance, a case of a very aggressive recurrent tumor that is slowed by therapies will be denoted as a positive outcome using RAI, whereas survival alone may not indicate a positive outcome. However, the RAI represents the integration of a number of factors, of which intrapatient biological variation is only one.

RAI is also a potential tool for counseling patients on potential risks and benefits of repeat surgery. It should be noted that the present results were obtained in a cohort of patients with optimally treated primary GBM, meaning that the tumor was nearly completely resected the last time and can be nearly completely resected this time as well. GBMs arising from a known, previously resected lowergrade tumor might have a different spectrum of potential RAIs. Time to progression after a previous resection that left a large residual tumor reflects factors other than tumor cell growth kinetics, especially the blood supply and size of the residual tumor. Large residual disease possibly responds differently to adjuvant therapies and could have a different mutation rate, both of which could impact the kinetics of recurrence after subsequent aggressive resection. Thus, the relative RAI outcomes after repeat surgery in these conditions deserve further analysis prior to using the RAI to counsel those patients.

TABLE 6. RAI stratified by adjuvant therapy

\begin{tabular}{cccccc}
\hline \multirow{2}{*}{ Variable } & \multicolumn{3}{c}{ Adjuvant Therapy } & & \\
\cline { 2 - 6 } & Temozolomide & Bevacizumab & Other Alkylating Agents & Other & None \\
\hline No. of patients & 43 & 3 & 11 & 5 & 34 \\
\hline RAl & & & & & \\
\hline$<1.0$ & $42 \%$ & $33 \%$ & $55 \%$ & $40 \%$ & $21 \%$ \\
\hline $1.0-2.0$ & $23 \%$ & $67 \%$ & $18 \%$ & $20 \%$ & $21 \%$ \\
\hline $2.0-5.0$ & $26 \%$ & $0 \%$ & $0 \%$ & $20 \%$ & $12 \%$ \\
\hline$>5.0$ & $9 \%$ & $0 \%$ & 0.82 & 2.17 & 1.23 \\
\hline Median RAl & 1.33 & 1.37 & & & \\
\hline
\end{tabular}


A limitation of our study, as in any study reporting surgical outcomes in glioma patients, is that there is an inherent selection bias for patients undergoing surgery. In general, many of the patients with the worst disease, either at presentation or, for the purposes of the present study, at recurrence, do not undergo surgery. Given this, we might expect the RAIs for the entire population of GBM patients to be higher than those seen in our study. In any case, our results address an important question: For patients undergoing repeat resection for focally recurrent GBM, is the progression-free interval after aggressive treatment a meaningful prognostic indicator for outcome? Our data suggest, somewhat counterintuitively, that it is not.

Another limitation of our study is a lack of qualityof-life measures. Unfortunately we did not collect these data prospectively in a usable way. Prolonging survival in patients with GBM is only relevant to the extent that quality of life is maintained to a significant degree, and future studies are warranted to assess quality of life longitudinally in patients over the course of multiple resections. Anecdotally, we have found that many patients undergoing repeated resections continue to do well and often maintain functional independence, although again we acknowledge the presence of selection bias in such an observation.

\section{Conclusions}

The present study is not an explicit attempt to advocate for or against repeat surgery for GBM, as there is no comparison cohort, and by own admission, there are patients who do well and others in whom the disease recurs very quickly. More importantly, we provide data regarding the spectrum of outcomes to counsel patients so they can make a decision of whether they wish to proceed. We demonstrate that the length of PFS provided by an aggressive repeat resection for primary GBM is highly variable and cannot be reliably predicted by the progression-free interval since the last surgery. This relationship likely results from multiple clinical and/or biological factors that are in need of further clarification. Importantly, the RAI might reflect the integrated effect of numerous known prognostic biomarkers, such as $M G M T$ methylation, $I D H-1$ mutation, etc., and these relationships deserve further clarification not possible in our dataset.

\section{References}

1. Berger MS, Deliganis AV, Dobbins J, Keles GE: The effect of extent of resection on recurrence in patients with low grade cerebral hemisphere gliomas. Cancer 74:1784-1791, 1994

2. Hemphill JC III, White DB: Clinical nihilism in neuroemergencies. Emerg Med Clin North Am 27:27-37, vii-viii, 2009

3. Kleihues P, Sobin LH: World Health Organization classification of tumors. Cancer 88:2887, 2000

4. Lacroix M, Abi-Said D, Fourney DR, Gokaslan ZL, Shi W, DeMonte F, et al: A multivariate analysis of 416 patients with glioblastoma multiforme: prognosis, extent of resection, and survival. J Neurosurg 95:190-198, 2001

5. Sanai N, Berger MS: Operative techniques for gliomas and the value of extent of resection. Neurotherapeutics 6:478486, 2009

6. Sanai N, Mirzadeh Z, Berger MS: Functional outcome after language mapping for glioma resection. N Engl J Med 358:18-27, 2008

7. Stummer W, Pichlmeier U, Meinel T, Wiestler OD, Zanella F, Reulen HJ: Fluorescence-guided surgery with 5-aminolevulinic acid for resection of malignant glioma: a randomised controlled multicentre phase III trial. Lancet Oncol 7:392401, 2006

8. Sughrue ME, Teo C: Minimally invasive intracranial surgery. Preface. Neurosurg Clin N Am 21:xi, 2010

9. Wen PY, Macdonald DR, Reardon DA, Cloughesy TF, Sorensen AG, Galanis E, et al: Updated response assessment criteria for high-grade gliomas: response assessment in neuro-oncology working group. J Clin Oncol 28:1963-1972, 2010

\section{Author Contributions}

Conception and design: Sughrue, Teo. Acquisition of data: Sughrue, Sheean. Analysis and interpretation of data: Sughrue, Sheean, Teo. Drafting the article: Sughrue, Sheean. Critically revising the article: Sughrue, Bonney, Maurer, Teo. Reviewed submitted version of manuscript: all authors. Approved the final version of the manuscript on behalf of all authors: Sughrue. Statistical analysis: Sughrue, Bonney. Administrative/technical/ material support: Bonney, Maurer.

\section{Correspondence}

Michael E. Sughrue, Department of Neurosurgery, University of Oklahoma Health Sciences Center, 1000 N. Lincoln Blvd., Ste. 4000, Oklahoma City, OK 73104. email: michael-sughrue@ ouhsc.edu. 